(2) Open Access Full Text Article

REVIEW

\title{
Improving outcomes in patients undergoing percutaneous coronary intervention: role of prasugrel
}

This article was published in the following Dove Press journal:

Vascular Health and Risk Management

20 May 2009

Number of times this article has been viewed

\section{Zuzana Motovska \\ Petr Widimsky \\ Third Medical Faculty of Charles University and University Hospital Kralovske Vinohrady, Prague, Czech Republic}

Correspondence: Zuzana Motovska 3rd Medical faculty of Charles University, Cardiocentre University Hospital Vinohrady, Srobarova 50, 10034 Prague 10, Czech Republic

Tel +42073 I 573253

Fax +42026716262I

Email zuzana.motovska@iex.cz

\begin{abstract}
Dual oral antiplatelet therapy, aspirin plus thienopyridine, has permitted a rapid increase in the use of coronary intervention procedures. Clopidogrel is the thienopyridine of choice for dual antiplatelet therapy in patients treated with percutaneous coronary intervention. However, there are two issues with clopidogrel: (1) clopidogrel's antiplatelet activity is delayed because the drug needs to be metabolized into its active form and (2) variability in patient response to clopidogrel has been demonstrated. To overcome these shortcomings of clopidogrel, new more potent inhibitors of $\mathrm{P} 2 \mathrm{Y} 12$ receptors, which have a more rapid onset of action have been introduced for clinical evaluation. This article is a nonexhaustive review of the literature and concentrates on prasugrel, a third-generation, oral thienopyridine. The purpose is to summarize the current knowledge about the benefits and risks of prasugrel and to outline the most prudent strategies for the drug's clinical use.
\end{abstract}

Keywords: P2Y12 receptors, prasugrel, oral thienopyridine, dual oral antiplatelet therapy

\section{Introduction}

Recognition that activation of platelets, rather than the coagulation pathway, increases the risk of stent-associated thrombosis ${ }^{1}$ has led to the strategy of platelet inhibition with a combination of antiplatelet drugs with complementary mechanisms of action as an adjuvant therapy for percutaneous coronary intervention (PCI). Complementary and independent mechanisms, irreversible inhibition of the thromboxane A2, adenosine diphosphate (ADP) and glycoprotein IIb/IIIa platelet recruitment pathways, have produced cumulative decreases in thrombotic events with acceptable bleeding risks following stent implantation. ${ }^{2}$

Dual oral antiplatelet therapy, aspirin plus thienopyridine, has permitted a rapid increase in the use of coronary intervention procedures. PCIs have become the most commonly performed coronary revascularization procedures, accounting for approximately $60 \%$ of all revascularizations. ${ }^{3,4}$ Therefore, optimizing the outcome after the procedure through the use of adjunctive antiplatelet therapy, which provides maximum protection against thrombosis without increasing the risk of bleeding, can have a substantial impact on cardiovascular morbidity and mortality.

\section{Thienopyridines}

Thienopyridine derivatives irreversibly modify platelet $\mathrm{P} 2 \mathrm{Y} 12$ receptors by covalently binding to cysteine residues of the receptor. ${ }^{5}$ The proportion of ADP receptors sensitive to the effects of thienopyridines is limited to $60 \%-70 \%{ }^{6}$ Currently there are two equally effective thienopyridines, ${ }^{7,8}$ ticlopidine and clopidogrel, available 
for clinical use. Clopidogrel is better tolerated and more convenient to use (once-daily dosing) compared to ticlopidine. $^{7}$ As a result, clopidogrel has almost replaced ticlopidine as the thienopyridine of choice for dual antiplatelet therapy in patients treated with PCI. ${ }^{9}$ Clopidogrel decreases the incidence of coronary stent thrombosis; additionally it has been approved and has proven beneficial in the reduction of myocardial infarction, stroke, and vascular death in patients with atherosclerotic vascular disease. ${ }^{10}$ Beyond its anti-aggregation effect, clopidogrel decreases the expression of activated platelet-dependent inflammatory markers such as the CD40 ligand (a potent stimulus of vascular inflammation) and CD62 P-selectin in patients undergoing PCI. ${ }^{11,12}$

\section{Clopidogrel limitations}

Clopidogrel is an inactive prodrug of thienopyridine, which needs to be metabolized by the hepatic cytochrome P450 enzymes (CYP450) into the active compound. ${ }^{13}$ However, only a small percentage of administered clopidogrel is metabolized by CYP450. The majority of clopidogrel is hydrolyzed to an inactive derivative that accounts for $85 \%$ of the clopidogrel-related compounds circulating in plasma. ${ }^{14}$ The need for metabolization delays the blocking of P2Y12 platelet receptors and thus, the drug's antiplatelet activity. Additionally, patient variability to clopidogrel has been demonstrated and shown to follow a typical bell-shaped or normal curve distribution. ${ }^{15,16}$ The variable inhibition of platelet aggregation (IPA) observed with clopidogrel seems to result from lower exposure to the active metabolite. ${ }^{17}$ Therefore, all factors that influence drug absorption ${ }^{18}$ and metabolic activation (ie, CYP450 activity) ${ }^{19,20}$ can affect drug effectiveness.

In the clopidogrel-efficacy curve, a relation between the pre- and post-treatment platelet reactivity index was found. ${ }^{21}$ Clopidogrel's capacity to inhibit platelet ADP-induced platelet activation was found to be limited. This can be partially explained by the greater proportion of low responders to clopidogrel in patients with diabetes who have enhanced platelet reactivity. ${ }^{20,22}$ Therefore, the degree of platelet suppression after clopidogrel was lower in patients undergoing PCI, for acute coronary syndrome, than in patients with stable coronary artery disease. ${ }^{21,23,24}$

Patients on clopidogrel therapy with lower responsiveness to clopidogrel had an increased rate of recurrent cardiovascular events..$^{25,26}$ The best antiplatelet effects occurred at loading doses of $600 \mathrm{mg}$ and maintenance doses of $150 \mathrm{mg}$ a day. ${ }^{27}$ Nonresponsiveness to high loading doses has also been reported in clinical studies. One of the largest of these studies
$(\mathrm{N}=804)$ reported that "nonresponsiveness" to a clopidogrel $600 \mathrm{mg}$ loading dose was a strong independent predictor of stent thrombosis in patients receiving drug-eluting stents. ${ }^{28}$

To overcome shortcomings of clopidogrel, new more potent inhibitors of $\mathrm{P} 2 \mathrm{Y} 12$ receptors, with a more rapid onset of action have been introduced for clinical evaluation, these include: prasugrel, cangrelor, and AZD 6140.

\section{Prasugrel Drug characteristics}

Prasugrel is also a prodrug and must be converted to an active form before binding irreversibly to the $\mathrm{P} 2 \mathrm{Y} 12$ receptor and inhibiting platelet aggregation for the life of the platelet. Prasugrel is rapidly absorbed and extensively metabolized. Prasugrel is quickly hydrolyzed to pharmacologically inactive thiolactone (R-95913). ${ }^{29,30}$ Thiolactone (R-95913) is further metabolized (oxidized) by intestinal and hepatic CYP-450 enzymes, which leads to formation of the active metabolite. In humans, renal excretion accounts for approximately $70 \%$ prasugrel metabolites. ${ }^{29,30}$ The antiplatelet effects of prasugrel are time- and dose-dependent.

\section{Onset of action and antiplatelet efficacy}

Conversion of clopidogrel to its active metabolite is a 2-step, CYP450-dependent process. ${ }^{14}$ However, prasugrel requires only one CYP450-dependent oxidative step to generate the thiol-containing active metabolite. ${ }^{30}$ This difference may underlie the more rapid metabolic conversion and onset of action of prasugrel compared with clopidogrel. ${ }^{31}$ The active metabolite of prasugrel was detected in plasma within $15 \mathrm{~min}$ of dosing and reached a maximum plasma concentration approximately $30 \mathrm{~min}$ after dosing. ${ }^{29,31}$

In a randomized, crossover, ex vivo study, healthy subjects received a single loading dose of prasugrel $(60 \mathrm{mg})$ or clopidogrel (300 mg). ${ }^{31}$ Inhibition of platelet aggregation with prasugrel was evident after $15 \mathrm{~min}$. Platelet inhibitory effects of a $60 \mathrm{mg}$ loading dose of prasugrel $30 \mathrm{~min}$ after administration was greater than the maximum antiplatelet effects of clopidogrel 12 hours after a $300 \mathrm{mg}$ loading dose.

In patients with stable atherosclerosis, prasugrel (60 mg loading dose) achieved quicker and greater inhibition of ADP-induced platelet aggregation, compared to a $600 \mathrm{mg}$ loading dose of clopidogrel. ${ }^{17}$ The difference in the IPA between clopidogrel and prasugrel was observed in as little as $30 \mathrm{~min}$ following drug administration. Within 30 minutes, prasugrel had achieved antiplatelet effects superior to the maximum antiplatelet effects attained by $600 \mathrm{mg}$ of clopidogrel over the 24-hour long observation period. 
The data from the PRINCIPLE-TIMI 44 (Prasugrel in Comparison to Clopidogrel for Inhibition of Platelet Activation and Aggregation- Thrombolysis in Myocardial Infarction $)^{32}$ study extend the results to patients undergoing cardiac catheterization for planned percutaneous coronary intervention. This trial compared the same dose regimen of prasugrel with high-dose clopidogrel (600 mg loading dose and $150 \mathrm{mg} / \mathrm{d}$ maintenance dose). Substantially and statistically significant greater platelet inhibition with prasugrel was observed at all time points studied during the loading dose and maintenance dose phases. Prasugrel was more potent and consistent in comparison to clopidogrel in the PCI setting.

The active metabolites of prasugrel and clopidogrel have similar potency at the platelet level. ${ }^{33}$ The greater potency of prasugrel to inhibit platelet $\mathrm{P} 2 \mathrm{Y} 12$ receptors in patients with coronary artery disease, compared with clopidogrel, has been linked to more efficient generation of prasugrel's active metabolite, to higher peak plasma levels and greater exposure of platelets to the active metabolite. ${ }^{17,31}$

\section{Inter-individual variability}

Studies including patients with coronary artery disease have documented inter-individual variability relative to clopidogrel's capacity to inhibit platelet aggregation. ${ }^{15,16,34}$ A substantial proportion $\left(24 \%{ }^{35}\right.$ nonresponders with the clopidogrel $300 \mathrm{mg}$ and $11 \%$ nonresponders with the clopidogrel $600 \mathrm{mg})^{34}$ of the patients undergoing elective PCI were evaluated as nonresponders to clopidogrel treatment. The proportion of patients, with limited clopidogrel efficacy, was even higher in patients with acute myocardial infarction. ${ }^{36}$ The percentage of prasugrel nonresponders, in patients with stable coronary artery disease (using the same definition for nonresponse; IPA $<20 \%$ in response to $20 \mu \mathrm{M}$ ADP), was only $3 \%$ after the 40 and $60 \mathrm{mg}$ doses of prasugrel. ${ }^{37}$ A crossover study in healthy, aspirin-free subjects demonstrated that all individuals who responded poorly to the clopidogrel $300 \mathrm{mg}$ achieved robust platelet inhibition when switched to prasugrel. ${ }^{31}$

\section{Translation of prasugrel benefits into the clinical outcomes}

The degree of suppression of platelet activity achieved through the use of antithrombotic agents should be balanced against the risk of atherothrombotic events. ${ }^{38}$ Accordingly the target population for prasugrel includes patients at high risk of thrombo-occlusive events:

- patients undergoing PCI for STE myocardial infarction,
- patients at risk of stent thrombosis and patients after stent thrombosis,

- diabetics undergoing PCI,

- patients with the presence of genetic variants related to nonresponsiveness to clopidogrel.

\section{Patients undergoing PCI for STE myocardial infarction}

The TRITON-TIMI 38 (TRial to assess Improvement in Therapeutic Outcomes by optimizing platelet inhibition with prasugrel $)^{39}$ found that in patients with acute coronary syndromes scheduled for PCI, prasugrel (60 mg loading dose and a $10 \mathrm{mg}$ daily maintenance dose), compared with approved doses of clopidogrel (300 mg loading dose and a $75 \mathrm{mg}$ daily maintenance dose), yielded significantly reduced rates of ischemic events. The primary efficacy end point (death from cardiovascular causes, nonfatal myocardial infarction, or nonfatal stroke) occurred in $12.1 \%$ of patients receiving clopidogrel and $9.9 \%$ of patients receiving prasugrel $(\mathrm{p}<0.001)$. There were also significant reductions in the prasugrel group relative to rates of (i) myocardial infarction $(9.7 \%$ for clopidogrel vs $7.4 \%$ for prasugrel; $\mathrm{p}<0.001$ ), (ii) urgent target-vessel revascularization $(3.7 \%$ vs $2.5 \%$; $<0.001)$, and (iii) stent thrombosis $(2.4 \%$ vs $1.1 \% ; \mathrm{p}<0.001)$. Death from cardiovascular causes and overall mortality did not differ significantly between treatment groups. However, this clinical benefit was accompanied by a significant increase in the risk of serious bleeding with prasugrel. Major bleeding was observed in $2.4 \%$ of patients receiving prasugrel and in $1.8 \%$ of patients receiving clopidogrel $(p=0.03)$. In addition, the prasugrel group showed an increased rate of life-threatening bleeding (1.4\% vs $0.9 \%$; $\mathrm{p}=0.01)$ and fatal bleeding $(0.4 \%$ vs $0.1 \% ; \mathrm{p}=0.002)$. A post hoc analysis of the study data identified three subgroups that had greater bleeding risk than the overall studied population: (i) patients with a history of stroke or transient ischemic attack, (ii) age $\geq 75$ years, and (iii) body weight $<60 \mathrm{~kg}$.

TRITON-TIMI 38 was designed to compare clopidogrel with prasugrel in patients undergoing PCI over the entire spectrum of acute coronary syndrome with moderate-tohigh-risks. Montalescot and colleagues ${ }^{40}$ recently published a prespecified analysis from the subgroup with STE myocardial infarctions. Patients with STE myocardial infarction underwent primary PCI within 12 hours of symptom onset or secondary PCI within 14 days of myocardial infarction for ongoing or recurrent ischemia or as part of a routine invasive strategy. ${ }^{39}$ The significant reduction in primary ischemic endpoint with prasugrel was consistent with that in the population with unstable angina or non-STE 
myocardial infarction. At 30 days, $6.5 \%$ of patients in the prasugrel arm had met the primary endpoint compared with $9.5 \%$ in the clopidogrel arm $(\mathrm{p}=0.0017)$. Cardiovascular death $(p=0.0469)$ and all causes of death $(p=0.0445)$ were also significantly reduced with prasugrel. After 15 months, rates of myocardial infarction and stent thrombosis remained significantly reduced with prasugrel. Differences in allcause and cardiovascular deaths between the groups lost statistical significance. The benefit from the more intensive platelet inhibitory effects associated with prasugrel was more pronounced in patients at higher risk. Patients with anterior myocardial infarctions had significantly $(\mathrm{p}=0.0003)$ lower incidence of primary endpoint with prasugrel $(9.8 \%)$ compared with clopidogrel (16.3\%). In individuals with nonanterior myocardial infarctions, treatment effects did not differ with regard to the primary endpoint $(9.9 \%$ clopidogrel vs $10.1 \%$ prasugrel; $p=0.8749$ ). In the subgroup with STE myocardial infarctions, no difference was reported between prasugrel and clopidogrel in thrombolysis in myocardial infarction (TIMI) major bleeding, life-threatening bleeding or intracranial hemorrhage. Prasugrel was associated with a significantly increased risk for TIMI major bleeding after coronary artery bypass graft surgery at 15 months, compared with clopidogrel (18.8\% vs $2.7 \%$; $=0.0033)$.

No randomized studies have been identified that compared prasugrel with clopidogrel $600 \mathrm{mg}$ in patients with acute coronary syndromes. The ongoing TRILOGY ACS (Targeted Platelet Inhibition to Clarify the Optimal Strategy to Medically Manage Acute Coronary Syndromes) study ${ }^{41}$ is comparing the tolerability and efficacy of a reduced dose of prasugrel (30 mg loading dose, $5 \mathrm{mg} / \mathrm{d}$ maintenance dose) with those of clopidogrel (300 mg loading dose, $75 \mathrm{mg} / \mathrm{d}$ maintenance dose) in reducing the risk of cardiovascular death, heart attack, or stroke in patients with an acute coronary syndrome who are to be medically managed without planned revascularization.

\section{Patients at risk of stent thrombosis and patients after stent thrombosis}

Despite combined antiplatelet therapy, stent thrombosis persists at a rate of $0.5 \%-2 \%$ in elective interventions, and up to $6 \%$ in patients with acute coronary syndromes. ${ }^{42}$ In cases of immediate reperfusion therapy by means of emergency PCI, patients with stent thrombosis have progressed to a major myocardial infarction, with a consequential significant decline in left ventricular function - a strong negative predictor of longterm survival. ${ }^{42-45} \mathrm{Clinical}$ and angiographic predictors of stent thrombosis have been identified; the most important predictors involved a poor post-procedural result such as inadequate stent expansion, residual dissection, and inappropriate IPA ${ }^{43,44}$ Therefore, improvement in stenting techniques and proper IPA represent variable of causal importance. Unfortunately, patient-related factors including diabetes mellitus, extent of coronary artery disease, and renal failure are not modifiable.

In the TRITON-TIMI 38, the overall mortality rate for patients with stent thrombosis was $26 \% .{ }^{45}$ In the same study, prasugrel, compared with the approved dose of clopidogrel, reduced the rate of stent thrombosis by $52 \%(\mathrm{p}<0.001)$. The reduction in stent thrombosis was consistent across multiple subgroups with respect to baseline characteristics (age, sex, acute coronary syndrome presentation, creatinine clearance, diabetes, or previous myocardial infarction) and treatment characteristics (glycoprotein IIb/IIIa use, stent length, and presence of bifurcation stenting). The greatest absolute benefits were seen in patients at higher risk for stent thrombosis, such as those with longer stents, bifurcation stents, impaired kidney function, and diabetes. Similar benefits of prasugrel were also seen in patients who received loading doses of study drugs before PCI $(0.83 \%$ vs $2.23 \% ; \mathrm{p}=0.002)$ or after the start of the procedure (1.24 vs $2.39 \%$; p $<0.0001)$.

Prasugrel reduced early $(1.56 \%$ vs $0.64 \% ; \mathrm{p}<0.001)$ as well as late $(0.82 \%$ vs $0.49 \% ; \mathrm{p}=0.035)$ stent thrombosis in patients with acute coronary syndromes treated with either bare metal or drug-eluting stents. ${ }^{45}$ As the TRITON results demonstrate, the reduction in stent thrombosis by prasugrel should be weighed against its increased bleeding risk. However, subgroup analyses of the study ${ }^{39,45}$ found no increased bleeding risk with prasugrel, compared with clopidogrel, in patients at increased risk of stent thrombosis, such as diabetic patients and those with STE myocardial infarction. Recommendation of prasugrel for patients at high risk of stent thrombosis and in patients after stent thrombosis would be a very prudent strategy.

\section{Diabetics undergoing $\mathbf{P C I}$}

Diabetes mellitus had an independent adverse effect on clinical outcomes in patients with coronary artery disease..$^{39,47,48}$ Diabetic patients have been shown to be poor responders to clopidogrel. ${ }^{20,49,50}$ Moreover, a high clopidogrel loading dose $(600 \mathrm{mg})$ has been unable to satisfactorily inhibit platelet reactivity in diabetics undergoing elective PCI. ${ }^{20,51}$ Prasugrel treatment (60 mg loading dose) resulted in a significantly lower proportion of diabetic patients with weak inhibition of ADP induced platelet activation, compared with $600 \mathrm{mg}$ of clopidogrel. ${ }^{51}$ The relationship between insufficient inhibition of platelet reactivity in diabetic patients, 
on dual antiplatelet therapy, and a greater risk of adverse cardiovascular events has been demonstrated. ${ }^{50}$

Subgroup analysis from the TRITON-TIMI $38^{52}$ reports that the composite of cardiovascular death, myocardial infarction, and stroke, was significantly reduced with prasugrel, among subjects without diabetes mellitus (9.2\% vs $10.6 \%$; $\mathrm{p}=0.02$ ), and even more significantly in patients with diabetes mellitus $(12.2 \%$ vs $17.0 \%$; $<<0.001)$, particularly in those taking insulin $(14.3 \%$ vs $22.2 \%$; $=0.009)$. Although TIMI major hemorrhage was increased among subjects without diabetes mellitus on prasugrel (2.4\%) vs clopidogrel (1.6\%); $\mathrm{p}=0.02)$, the rates were similar among subjects with diabetes mellitus $(2.6 \%$ vs $2.5 \% ; \mathrm{p}=0.81)$. In diabetic patients, the rate of TIMI major or minor bleeding was observed in $5.3 \%$ with prasugrel and $4.3 \%$ with clopidogrel $(\mathrm{p}=0.13)$. Therefore, the net clinical benefit with prasugrel was greater for patients with diabetes than for patients without diabetes ( $8 \%$ vs $26 \%$ ). The greater platelet inhibition among patients with diabetes results in improved outcomes.

\section{Patients with genetic variants related to nonresponsiveness to clopidogrel}

Focusing on the relationship between genetics and therapy efficacy, could provide clinical advances, mostly for drugs with observed high inter-individual efficacy variability and life-threatening consequences resulting from inefficacy of these drugs. From this point of view, clopidogrel is an ideal candidate for pharmacogenetic studies which target the genetics behind clopidogrel inefficacy.

A significant reduction in clopidogrel antiplatelet effectiveness, linked to the P1A2 polymorphism, has been found. A $600 \mathrm{mg}$ loading dose of clopidogrel failed to acceptably inhibit platelet reactivity in patients with stable coronary artery disease undergoing elective PCI who were positive for this polymorphism. ${ }^{53}$

Genetic variants of CYP450 have been linked to a reduced exposure to the active drug metabolite, less platelet inhibition, and less protection from recurrent ischemic events in persons receiving clopidogrel. ${ }^{54,55}$ In patients with acute coronary syndromes treated with clopidogrel, the presence of CYP 2C19 reduced-function allele was associated with adverse clinical outcomes, including an increased death rate from cardiovascular causes, myocardial infarction, or stroke, especially among patients undergoing PCI. ${ }^{54,55}$ Notably, the rate of stent thrombosis was three times greater than that among noncarriers. ${ }^{55}$
The contribution of genetic testing relative to routine clinical practice remains unclear. Therefore, CYP 2C19 reduced-function allele carriers are an optimal target population for the study of new antithrombotic regimens, which includes prasugrel, the efficacy of which is unaffected by variability in CYP2C19 isoenzymes. ${ }^{56}$

\section{Conclusion}

The degree of suppression of platelet activity achieved through the use of antithrombotic agents should be balanced against the risk of atherothrombotic events. Prasugrel, in comparison to clopidogrel, is a more potent inhibitor of P2Y12 receptors, and has a more rapid onset of action. Accordingly the target population for prasugrel should include patients at high risk of thrombo-occlusive events, eg, patients undergoing percutaneous coronary intervention for STE myocardial infarction, patients at risk of stent thrombosis or patients after stent thrombosis, diabetics undergoing PCI, or patients with the presence of genetic variants related to nonresponsiveness to clopidogrel.

The Committee for Medicinal Products for Human Use of the European Medicines Agency issued a positive opinion and recommended approval of prasugrel. Thereafter, the European Commission granted marketing approval of prasugrel (Efient, Lilly/Daiichi Sankyo) for the prevention of atherothrombotic events in patients with acute coronary syndromes undergoing PCI. ${ }^{57}$ Prasugrel will be marketed in European countries as early as April 2009. The initial, worldwide, launch of prasugrel took place in the UK. A positive nod from the US Food and Drug Administration is expected soon. ${ }^{58}$

\section{Acknowledgments}

This study was supported by the Charles University Prague Research Project number MSM0021620817 and by Research Project number MZ0VFN2005 Ministry of Health, Czech Republic.

\section{Disclosure}

The authors do not have any financial or personal relationships that could inappropriately influence (or bias) the authors' decisions, work, or manuscript.

\section{References}

1. Neumann FJ, Gawaz M, Ott I, May A, Mössmer G, Schömig A. Prospective evaluation of hemostatic predictors of subacute stent thrombosis after coronary Palmaz-Schatz stenting. J Am Coll Cardiol. 1996;27:15-21.

2. Makkar RR, Eigler S, Kaul S, et al. Effects of clopidogrel, aspirin and combined therapy in a porcine ex vivo model of high-shear induced stent thrombosis. Eur Heart J. 1998;19:1538-1546. 
3. Lenzen MJ, Boersma E, Bertrand ME, et al; European Society of Cardiology. Management and outcome of patients with established coronary artery disease: the Euro Heart Survey on coronary revascularization. Eur Heart J. 2005;26(12):1169-1179.

4. Thom T, Haase N, Rosamond W, et al; American Heart Association Statistics Committee and Stroke Statistics Subcommittee. Heart disease and stroke statistics - 2006 update: a report from the American Heart Association Statistics Committee and Stroke Statistics Subcommittee. Circulation. 2006;113(6):e85-e101.

5. Savi P, Pereillo JM, Uzabiaga MF, et al. Identification and biological activity of the active metabolite of clopidogrel. Thromb Haemost. 2000; 84:891-896.

6. Ibanez B, Vilahur G, Badimon JJ, Pharmacology of thienopyridines: rationale for dual pathway inhibition. Eur Heart J Suppl. 2006;8: G3-G9.

7. Bertrand ME, Rupprecht HJ, Urban P, Gershlick AH. Double-blind study of the safety of clopidogrel with and without a loading dose in combination with aspirin compared with ticlopidine in combination with aspirin after coronary stenting: the Clopidogrel Aspirin Stent International Cooperative Study (CLASSICS). Circulation. 2000;102:624-629.

8. Bhatt DL, Bertrand ME, Berger PB, et al. Meta-analysis of randomized and registry comparisons of ticlopidine and clopidogrel after stenting. J Am Coll Cardiol. 2002;39:9-14.

9. Silber S, Albertsson P, Aviles FF, et al; for Task Force for Percutaneous Coronary Interventions of the European Society of Cardiology. Guidelines for percutaneous coronary interventions. The Task Force for Percutaneous Coronary Interventions of the European Society of Cardiology. Eur Heart J. 2005;26(8):804-847.

10. Eshaghian S, Kaul S, Amin S, Shah PK, Diamond GA. Role of clopidogrel in managing atherothrombotic cardiovascular disease. Ann Intern Med. 2007;146(6):434-441.

11. Quinn MJ, Bhatt DL, Zidar F, et al. Effect of clopidogrel pretreatment on inflammatory marker expression in patients undergoing percutaneous coronary intervention. Am J Cardiol. 2004;93:679-684.

12. Vivekananthan DP, Bhatt DL, Chew DP, et al. Effect of clopidogrel pretreatment on periprocedural rise in C-reactive protein after percutaneous coronary intervention. Am J Cardiol. 2004;94:358-360.

13. Pereillo JM, Maftouh M, Andrieu A, et al. Structure and stereochemistry of the active metabolite of clopidogrel. Drug Metab Dispos. 2002;30: $1288-1295$

14. Clarke TA, Waskell LA. The metabolism of clopidogrel is catalyzed by human cytochrome P450 3A and is inhibited by atorvastatin. Drug Metab Dispos. 2003;31:53-59.

15. Gurbel PA, Bliden KP, Hiatt BL, O'Connor CM. Clopidogrel for coronary stenting: response variability, drug resistance, and the effect of pretreatment platelet reactivity. Circulation. 2003;107:2908-2913.

16. Serebruany VL, Steinhubl SR, Berger PB, Malinin AI, Bhatt DL, Topol EJ. Variability in platelet responsiveness to clopidogrel among 544 individuals. J Am Coll Cardiol. 2005;45:246-251.

17. Wallentin L, Varenhorst C, James S, et al. Prasugrel achieves greater and faster $\mathrm{P} 2 \mathrm{Y}_{12}$ receptor-mediated platelet inhibition than clopidogrel due to more efficient generation of its active metabolite in aspirin-treated patients with coronary artery disease. Eur Heart J. 2008;29:21-30.

18. Taubert D, von Beckerath N, Grimberg G, et al. Impact of P-glycoprotein on clopidogrel absorption. Clin Pharmacol Ther. 2006;80:486-501.

19. Simon T, Verstuyft C, Mary-Krause M, et al. Genetic determinants of response to clopidogrel and cardiovascular events. N Engl J Med. 2009;360:363-375.

20. Motovska Z, Widimsky P, Petr R, et al. on behalf of the PRAGUE-8 Study Investigators. Factors influencing clopidogrel efficacy in patients with stable coronary artery disease undergoing elective percutaneous coronary intervention: statin's advantage and the smoking "paradox". $J$ Cardiovasc Pharmacol. 2009 Feb 25. [Epub ahead of print].

21. Motovska Z, Widimsky P, Petr R, et al. Optimal pretreatment timing for high load dosing $(600 \mathrm{mg})$ of clopidogrel before planned percutaneous coronary intervention for maximal antiplatelet effectiveness. Int J Cardiol. $2009 \mathrm{Feb}$ 14. [Epub ahead of print].
22. Davì G, Gresele P, Violi F, et al. Diabetes mellitus, hypercholesterolemia, and hypertension but not vascular disease per se are associated with persistent platelet activation in vivo. Evidence derived from the study of peripheral arterial disease. Circulation. 1997;96(1):69-75.

23. Soffer D, Moussa I, Harjai K, et al. Impact of angina class on inhibition of platelet aggregation following clopidogrel loading in patients undergoing coronary intervention: do we need more aggressive dosing regimens in unstable angina? Catheter Cardiovasc Interv. 2003;59:21-25.

24. Montalescot G, Sideris G, Meuleman C, et al; for the ALBION Trial Investigators. A randomized comparison of high clopidogrel loading doses in patients with non-ST-segment elevation acute coronary syndromes: the ALBION (Assessment of the Best Loading Dose of Clopidogrel to Blunt Platelet Activation, Inflammation and Ongoing Necrosis) trial. J Am Coll Cardiol. 2006;48:931-938.

25. Geisler T, Langer H, Wydymus M, et al. Low response to clopidogrel is associated with cardiovascular outcome after coronary stent implantation. Eur Heart J. 2006;27(20):2420-2425.

26. Angiolillo DJ, Bernardo E, Sabate M, et al. Impact of platelet reactivity on cardiovascular outcomes in patients with type 2 diabetes mellitus and coronary artery disease $J$ Am Coll Cardiol. 2007;50:1541-1547.

27. von Beckerath N, Taubert D, Pogatsa-Murray G, Schomig E, Kastrati A, Schomig A. Absorption, metabolization, and antiplatelet effects of 300-, 600-, and 900-mg loading doses of clopidogrel: Results of the ISAR-CHOICE (Intracoronary Stenting and Antithrombotic Regimen: Choose Between 3 High Oral Doses for Immediate Clopidogrel Effect) Trial. Circulation. 2005;112(19):2946-2950.

28. Buonamici P, Marcucci R, Migliorini A, et al. Impact of platelet reactivity after clopidogrel administration on drug-eluting stent thrombosis. J Am Coll Cardiol. 2007;49(24):2312-2317.

29. Farid NA, Smith RL, Gillespie TA, et al. The disposition of prasugrel, a novel thienopyridine, in humans. Drug Metab Dispos. 2007;35: 1096-1104.

30. Jakubowski JA, Winters KJ, Naganuma H, Wallentin L. Prasugrel: A novel thienopyridine antiplatelet agent. Cardiovasc Drug Rev. 2007; 25(4):357-374.

31. Brandt JT, Payne CD, Wiviott SD, et al. A comparison of prasugrel and clopidogrel loading doses on platelet function: Magnitude of platelet inhibition is related to active metabolite formation. Am Heart $J$. 2007;153:e9-e16.

32. Wiviott SD, Trenk D, Frelinger AL, et al. Prasugrel compared with high loading- and maintenance-dose clopidogrel in patients with planned percutaneous coronary intervention: the Prasugrel in Comparison to Clopidogrel for Inhibition of Platelet Activation and AggregationThrombolysis in Myocardial Infarction 44 trial. Circulation. 2007;116: 2923-2932.

33. Sugidachi A, Ogawa T, Kurihara A, et al. The greater in vivo antiplatelet effects of prasugrel as compared to clopidogrel reflect more efficient generation of its active metabolite with similar antiplatelet activity to that of clopidogrel's active metabolite. J Thromb Haemost. 2007;5:1545-1551.

34. Muller I, Besta F, Schulz C, Massberg S, Schomig A, Gawaz M. Prevalence of clopidogrel non-responders among patients with stable angina pectoris scheduled for elective coronary stent placement. Thromb Haemost. 2003;89:783-787.

35. Lev EI, Patel RT, Maresh KJ, et al. Aspirin and clopidogrel drug response in patients undergoing percutaneous coronary intervention: the role of dual drug resistance. J Am Coll Cardiol. 2006;47(1):27-33.

36. Matetzky S, Shenkman B, Guetta V, et al. Clopidogrel resistance is associated with increased risk of recurrent atherothrombotic events in patients with acute myocardial infarction. Circulation. 2004;109:3171-3175.

37. Jernberg T, Payne CD, Winters KJ, et al. Prasugrel achieves greater inhibition of platelet aggregation and a lower rate of non-responders compared with clopidogrel in aspirin-treated patients with stable coronary artery disease. Eur Heart J. 2006;27:1166-1173.

38. Motovska Z, Kala P. Benefits and risks of clopidogrel use in patients with coronary artery disease: Evidence from randomized studies and registries. Clin Ther. 2008;30P2:2191-2202. 
39. Wiviott SD, Braunwald E, McCabe CH, et al; TRITON-TIMI 38 Investigators. Prasugrel versus clopidogrel in patients with acute coronary syndromes. $N$ Eng J Med. 2007;357:2001-2015.

40. Montalescot G, Wiviott SD, Braunwald E, Murphy SA, Gibson CM, McCabe CH, Antman EM; TRITON-TIMI 38 investigators. Prasugrel compared with clopidogrel in patients undergoing percutaneous coronary intervention for ST-elevation myocardial infarction (TRITON-TIMI 38): double-blind, randomised controlled trial. Lancet. 2009;373(9665):723-731.

41. US National Institutes of Health. A comparison of prasugrel and clopidogrel in acute coronary syndrome subjects (TRILOGY ACS). April 20, 2009. Accessed on May 6, 2009. Available from: http://clinicaltrials. gov/ct2/show/NCT00699998.

42. Wenaweser P, Rey C, Eberli F, et al. Stent thrombosis following bare-metal stent implantation success of emergency percutaneous coronary intervention and predictors of adverse outcome. Eur Heart J. 2005;26(12):1180-1187.

43. Cook S, Windecker S. Early stent thrombosis: Past, present, and future. Circulation. 2009; 119:657-659.

44. Iakovou I, Schmidt T, Bonizzoni E, et al. Incidence, predictors, and outcome of thrombosis after successful implantation of drug-eluting stents. JAMA. 2005;293:2126-2130.

45. Wiviott $\mathrm{SD}$, Braunwald $\mathrm{E}, \mathrm{McCabe} \mathrm{CH}$, et al. Intensive oral antiplatelet therapy for reduction of ischaemic events including stent thrombosis in patients with acute coronary syndromes treated with percutaneous coronary intervention and stenting in the TRITON-TIMI 38 trial: a subanalysis of a randomised trial. Lancet. 2008;371(9621):1353-1363.

46. Wiviott SD, Braunwald E, Angiolillo DJ, et al; TRITON-TIMI 38 Investigators. Greater clinical benefit of more intensive oral antiplatelet therapy with prasugrel in patients with diabetes mellitus in the trial to assess improvement in therapeutic outcomes by optimizing platelet inhibition with prasugrel - Thrombolysis in Myocardial Infarction 38. Circulation. 2008;118(16):1626-1636.

47. Donahoe SM, Stewart GC, McCabe CH, et al. Diabetes and mortality following acute coronary syndromes. JAMA. 2007;298:765-775.

48. Yusuf S, Zhao F, Mehta SR, Chrolavicius S, Tognoni G, Fox KK. Effects of clopidogrel in addition to aspirin in patients with acute coronary syndromes without ST-segment elevation. $N$ Engl $J$ Med. 2001;345:494-502.
49. Angiolillo DJ, Showmaker SB, Desai B, et al. Randomized comparison of a high clopidogrel maintenance dose in patients with diabetes mellitus and coronary artery disease: results of the optimizing antiplatelet therapy in diabetes mellitus (OPTIMUS) study. Circulation. 2007;115(6):708-716.

50. Angiolillo DJ, Bernardo E, Sabate M, et al. Impact of platelet reactivity on cardiovascular outcomes in patients with type 2 diabetes mellitus and coronary artery disease $J$ Am Coll Cardiol. 2007;50:1541-1547.

51. Erlinge D, Varenhorst C, Braun OÖ, et al. Patients with poor responsiveness to thienopyridine treatment or with diabetes have lower levels of circulating active metabolite, but their platelets respond normally to active metabolite added ex vivo. $J$ Am Coll Cardiol. 2008;52:1968-1977.

52. Wiviott SD, Braunwald E, Angiolillo DJ, et al. Greater clinical benefit of more intensive oral antiplatelet therapy with prasugrel in patients with diabetes mellitus in TRITON-TIMI 38. Circulation. 2008;118:1626-1636.

53. Motovska Z, Widimsky P, Kvasnicka J, et al; on behalf of the PRAGUE-8 study investigators. High loading dose of clopidogrel is unable to satisfactorily inhibit platelet reactivity in patients with glycoprotein IIIA gene polymorphism - a genetic substudy of PRAGUE-8 trial. Blood Coagul Fibrin. 2009. March 16; doi:10.1097/ MBC.0b013e328325455b

54. Simon T, Verstuyft C, Mary-Krause M, et al. Genetic determinants of response to clopidogrel and cardiovascular events. $N$ Engl J Med. 2009;360:363-375.

55. Mega JL, Close SL, Wiviott SD, et al. Cytochrome P-450 polymorphisms and response to clopidogrel. $N$ Engl J Med. 2009;360:354-362.

56. Brandt JT, Close SL, Iturria SJ, et al. Common polymorphisms of CYP2C19 and CYP2C9 affect the pharmacokinetic and pharmacodynamic response to clopidogrel but not prasugrel. J Thromb Haemost. 2007;5:2429-2436.

57. Eli Lilly and Company. European Commission approves EFIENT (prasugrel) for patients with acute coronary syndrome undergoing PCI [press release]. February 23, 2009. Accessed on May 6, 2009. Available from: http://newsroom.lilly.com/releasedetail.cfm?ReleaseID=366955.

58. Eli Lilly and Company. Prasugrel receives unanimous approval recommendation from FDA Advisory Committee. February 3, 3009 Accessed on May 6, 2009. Available from: http://www.drugs.com/nda/ effient_090204.html.
Vascular Health and Risk Management

\section{Publish your work in this journal}

Vascular Health and Risk Management is an international, peerreviewed journal of therapeutics and risk management, focusing on concise rapid reporting of clinical studies on the processes involved in the maintenance of vascular health; the monitoring, prevention and treatment of vascular disease and its sequelae; and the involvement of

\section{Dovepress}

metabolic disorders, particularly diabetes. This journal is indexed on PubMed Central and MedLine. The manuscript management system is completely online and includes a very quick and fair peer-review system, which is all easy to use. Visit http://www.dovepress.com/ testimonials.php to read real quotes from published authors. 